\title{
Shifting attention does not influence numerical processing
}

\author{
Andrew Clement ${ }^{1,2} \cdot$ Alexandra Moffat $^{1} \cdot$ Jay Pratt $^{1}$
}

Published online: 10 September 2020

(C) The Psychonomic Society, Inc. 2020

\begin{abstract}
Many theories of numerical cognition assume that numbers and space share a common representation at the response level. For example, observers are faster to respond to small numbers with their left hand and large numbers with their right hand (the SNARC effect). There is also evidence that viewing numbers can produce spatial shifts of attention, suggesting that attention may play a role in the spatial representation of numbers. In the present study, we assessed whether shifts of attention can influence numerical processing. Participants viewed a leftward or rightward peripheral cue followed by a centrally presented number, then judged whether the number was odd or even. Participants responded faster and made fewer errors when the number magnitude and response side were compatible, revealing a response-based SNARC effect. Participants also responded faster when the cue direction and response side were compatible, revealing a Simon effect. However, participants did not respond faster when the cue direction and number magnitude were compatible. Similar findings were observed when the association between numbers and space was relatively explicit. Moreover, although we failed to observe a response-based SNARC effect when number magnitude was directly relevant to observers' task, we observed a large Simon effect. Together, these findings suggest that although numbers and space share a common representation at the response level, attention does not play a substantial role in the spatial representation of numbers.
\end{abstract}

Keywords Numbers $\cdot$ Spatial processing $\cdot$ Attention $\cdot$ Spatial cueing $\cdot$ Embodied cognition

Many theories of numerical cognition assume that numbers and space share a common representation. This association between numbers and space is not only reflected in the common use of graphs and number lines, but can also influence the processing of numerical information. An influential example of this comes from a study by Dehaene, Bossini, and Giraux (1993). In their study, participants viewed a centrally presented number, then judged whether the number was odd or even. The researchers found that participants were faster to respond to small numbers with their left hand and large numbers with their right hand (see also Dehaene, Dupoux, \& Mehler, 1990). This spatialnumerical association of response codes (the SNARC effect) suggests that observers represent numerical information along a mental number line, with small numbers on the left and large numbers on the right. Importantly, the SNARC effect has been

Andrew Clement

andrew.clement@tamu.edu

1 Department of Psychology, University of Toronto, Toronto, ON M5S 1A1, Canada

2 Department of Psychological \& Brain Sciences, Texas A\&M University, College Station, TX 77843, USA observed for both parity and magnitude judgments (Dehaene et al., 1993; Dehaene et al., 1990), suggesting that this effect can occur regardless of whether number magnitude is relevant to observers' task. Moreover, this effect has been replicated using saccadic responses (Fischer, Warlop, Hill, \& Fias, 2004; Schwarz \& Keus, 2004), pointing responses (Fischer, 2003), and responses with observers' hands and feet (Schwarz $\&$ Müller, 2006). Together, these findings suggest that numbers and space share a common representation at the response level.

Although many theoretical models suggest that the SNARC effect occurs at the response selection stage (Daar \& Pratt, 2008; Gevers, Caessens, \& Fias, 2005; Gevers, Verguts, Reynvoet, Caessens, \& Fias, 2006; Keus \& Schwarz, 2005; Keus, Jenks, \& Schwarz, 2005), there is also evidence that viewing numbers can influence the spatial allocation of attention. For example, Fischer, Castel, Dodd, and Pratt (2003) had participants view a centrally presented number, then asked them to detect a leftward or rightward target in a spatial cueing task. The researchers found that small numbers facilitated the detection of leftward targets and large numbers facilitated the detection of rightward targets. This attentional SNARC effect not only demonstrates that numbers can act as spatial cues for attention, but also suggests that viewing 
numbers can produce spatial shifts of attention. Consistent with this suggestion, several studies have found that viewing numbers can produce event-related potentials (ERPs) associated with early sensory processing and spatial shifts of attention (Ranzini, Dehaene, Piazza, \& Hubbard, 2009; Salillas, El Yagoubi, \& Semenza, 2008; Schuller, Hoffman, Goffaux, \& Schiltz, 2015).

The notion that attention may play a role in the spatial representation of numbers is also supported by neurophysiological evidence. For example, the representation of numbers and space rely on many of the same areas in posterior parietal cortex (Dehaene, Piazza, Pinel, \& Cohen, 2003; Hubbard, Piazza, Pinel, \& Dehaene, 2005). Critically, these areas are also involved in the programming of eye movements and other shifts of attention (e.g., Colby \& Goldberg, 1999). Patients with right parietal damage also exhibit a pattern of hemispatial neglect, in which they fail to attend to stimuli in the contralateral visual hemifield. Importantly, these patients not only misplace the midpoint of a line in a line bisection task, but also show a similar pattern of errors when judging the midpoint of a numerical interval (Zorzi, Priftis, \& Umiltà, 2002). Together, these findings provide further evidence that attention may play a role in the spatial representation of numbers. Indeed, several influential theories of numerical cognition have proposed that shifts of attention may underlie the processing of numerical information (Dehaene et al., 2003; Hubbard et al., 2005).

Although the previous findings suggest that viewing numbers can produce spatial shifts of attention, a growing body of research has questioned the reliability of these effects. For example, while some studies have replicated the attentional SNARC effect (Dodd, Van der Stigchel, Leghari, Fung, \& Kingstone, 2008; Galfano, Rusconi, \& Umiltà, 2006; He et al., 2020; Ristic, Wright, \& Kingstone, 2006), other studies have either failed to replicate this effect (Bonato, Priftis, Marenzi, \& Zorzi, 2009; Colling et al., 2020; Pellegrino et al., 2019) or have only replicated this effect when the association between numbers and space is relatively explicit (Fattorini, Pinto, Rotondaro, \& Doricchi, 2015; Pinto et al., 2018; Ristic et al., 2006) or when number magnitude is directly relevant to observers' task (Casarotti, Michielin, Zorzi, \& Umiltà, 2007; Zanolie \& Pecher, 2014). Other studies have found that viewing numbers can produce ERPs associated with spatial shifts of attention, but have failed to observe behavioral evidence for the attentional SNARC effect (Ranzini et al., 2009; Salillas et al., 2008; Schuller et al., 2015). Lastly, several studies have found that the attentional SNARC effect can be reversed using different task instructions (Galfano et al., 2006; Ristic et al., 2006). Together, these findings suggest that viewing numbers does not automatically produce spatial shifts of attention.

While a growing body of research has questioned the notion that viewing numbers can produce spatial shifts of attention, some theoretical models suggest that numbers and space share a common representation at the response level (Daar \& Pratt, 2008; Gevers et al., 2005; Gevers et al., 2006; Keus \& Schwarz, 2005; Keus et al., 2005). For example, several studies have found that the SNARC effect interacts with the Simon effect (Gevers et al., 2005; Keus \& Schwarz, 2005; but see Mapelli, Rusconi, \& Umiltà, 2003), in which participants are faster to respond to leftward stimuli with their left hand and rightward stimuli with their right hand (Simon, 1969). Because the Simon effect is thought to occur at the response selection stage, this suggests that the SNARC effect occurs at a similar processing stage. Fattorini et al. (2015) also directly compared the SNARC and attentional SNARC effects and found that these effects did not interact with one another. Moreover, while the researchers consistently replicated the SNARC effect, they only replicated the attentional SNARC effect when participants were asked to imagine the position of a number along the mental number line (see also Pinto et al., 2018).

If viewing numbers does not automatically produce spatial shifts of attention, it is also possible that attention may not play a substantial role in the spatial representation of numbers. To test whether this is the case, we investigated the broader claim that shifts of attention underlie the processing of numerical information. While some studies have assessed whether viewing numbers can produce spatial shifts of attention, few studies have assessed whether shifts of attention can influence numerical processing. However, a recent study by Mills, Boychuk, Chasteen, and Pratt (2018) suggests that shifts of attention can influence the processing of other stimuli with implicit spatial associations. In their study, participants viewed an upward or downward peripheral cue followed by a centrally presented letter string, then judged whether the string was a word or nonword. In this case, upward cues facilitated the processing of words with upward spatial associations (e.g., God, hat) and downward cues facilitated the processing of words with downward spatial associations (e.g., Devil, boots). Because these cues are thought to automatically produce spatial shifts of attention (e.g., Posner, 1980), these findings suggest that shifts of attention can influence language processing. In the present study, we used a similar method to assess whether shifts of attention can influence numerical processing.

\section{Experiment 1}

To assess whether shifts of attention can influence numerical processing, we had participants view a leftward or rightward peripheral cue followed by a centrally presented number, then asked them to judge whether the number was odd or even. If attention plays a role in the spatial representation of numbers, leftward cues should facilitate the processing of small 
numbers and rightward cues should facilitate the processing of large numbers. In addition to assessing whether shifts of attention can influence numerical processing, this method allowed us to test whether numbers and space share a common representation at the response level. If this is the case, small numbers should facilitate leftward responses and large numbers should facilitate rightward responses.

Although many studies use a relatively small set of onedigit numbers, in the present study we used a larger set of twodigit numbers. We selected this method for two reasons. First, this method replicates early demonstrations of the SNARC effect, in which participants judged the parity or magnitude of two-digit numbers (Dehaene et al., 1993; Dehaene et al., 1990). Second, some researchers have suggested that the attentional SNARC effect may not be due to shifts of attention, but instead may arise from the spatial correspondence between numbers and spatial locations (e.g., Proctor \& Cho, 2006). According to this view, using a relatively small set of numbers may create task-relevant associations between numbers and space, which may account for the attentional SNARC effect. By using a larger set of two-digit numbers, we sought to ensure that any effects were due to shifts of attention rather than the spatial correspondence between numbers and spatial locations.

\section{Method}

Participants In a previous replication study of the attentional SNARC effect, data were collected from a total of 20 participants in a within-subjects design (Zanolie \& Pecher, 2014). However, because several studies have failed to replicate this effect, we increased our sample size to 40 participants. Assuming a small effect size $(f=0.1)$ and a moderate correlation between levels of our within-subjects variables $(\rho=$ 0.5 ), an a priori power analysis conducted using $\mathrm{G}^{*}$ Power 3 (Faul, Erdfelder, Lang, \& Buchner, 2007) indicated that this sample size would be sufficient to detect a three-way interaction between response mapping, cue direction, and number magnitude at $80 \%$ statistical power. As a result, a group of 42 University of Toronto undergraduates (33 females; mean age $=19.1$ years) participated for $\$ 10$ or course credit. All participants were right-handed and were native English speakers. Two participants were excluded because they made a large proportion of errors ( $\sim 35 \%$ or greater).

Apparatus and stimuli Stimuli consisted of a series of twodigit numbers between 11 and 99 . Half of the numbers were small $(11,12,13,14,21,22,23,24)$ and the other half were large $(86,87,88,89,96,97,98,99)$. Because observers rely on both digits when judging the parity or magnitude of twodigit numbers (e.g., Nuerk, Weger, \& Willmes, 2001), numbers were selected so that both digits were smaller or larger than 5. Each number was presented in 40-point Arial font, and subtended $0.8^{\circ}$ vertically. All stimuli were presented in gray $\left(45.6 \mathrm{~cd} / \mathrm{m}^{2}\right)$ on a black background $\left(<0.01 \mathrm{~cd} / \mathrm{m}^{2}\right)$. Stimuli were presented on a 24-in LCD monitor with a refresh rate of $60 \mathrm{~Hz}$. Participants sat $57 \mathrm{~cm}$ from the monitor so that it subtended $53.3^{\circ}$ horizontally and $30^{\circ}$ vertically.

Procedure and design At the beginning of each trial, a gray fixation cross $\left(45.6 \mathrm{~cd} / \mathrm{m}^{2} ; 1^{\circ} \times 1^{\circ}\right)$ appeared in the center of the screen, flanked by two gray placeholders $\left(45.6 \mathrm{~cd} / \mathrm{m}^{2} ; 4^{\circ} \times\right.$ $4^{\circ}$; see Fig. 1). The placeholders were $0.04^{\circ}$ in thickness, and were presented $8^{\circ}$ to the left and right of fixation. After a randomly determined interval between 800 and $1,200 \mathrm{~ms}$, a white peripheral cue $\left(96.5 \mathrm{~cd} / \mathrm{m}^{2} ; 4^{\circ} \times 4^{\circ}\right)$ was presented at the location of one of these placeholders. This cue was $0.32^{\circ}$ in thickness, and was presented $8^{\circ}$ to the left or right of fixation. After $100 \mathrm{~ms}$, the display was cleared, and a two-digit number was presented in the center of the screen. Using the index and middle finger of their right hand, participants pressed the " $n$ " or " $m$ " key to identify whether the number was odd or even. Participants received an error message if they responded incorrectly or if their response times were less than $100 \mathrm{~ms}$ or greater than $2,000 \mathrm{~ms}$.

Participants completed three blocks of 160 trials, for a total of 480 trials. Each number was presented randomly and equally often within a block, and was preceded equally often by a leftward or rightward cue. As a result, the cue direction and the magnitude of the numbers were counterbalanced across trials. The mapping of the response keys was also counterbalanced across participants. As a result, the response side could be either compatible or incompatible with the magnitude of the numbers. Compatible response mappings were defined as leftward responses to small numbers and rightward responses to large numbers, while incompatible response mappings were defined as rightward responses to small numbers and leftward responses to large numbers.

\section{Results}

Incorrect responses and response times less than $100 \mathrm{~ms}$ or greater than 2,000 ms were excluded from analysis. To test whether shifts of attention influence parity judgments, we analyzed average response times using a 2 (response mapping: compatible, incompatible) $\times 2$ (cue direction: left, right $) \times 2$ (number magnitude: small, large) repeated-measures analysis of variance (ANOVA). The analysis revealed a significant main effect of response mapping, $F(1,39)=43.70, p<.001, \eta_{\mathrm{p}}{ }^{2}=$ .528 , with participants responding faster when the number magnitude and response side were compatible $(M=593.53 \mathrm{~ms}, S D$ $=93.55 \mathrm{~ms})$ than when they were incompatible $(M=617.89$ $\mathrm{ms}, S D=96.08 \mathrm{~ms}$ ). Thus, we observed a response-based SNARC effect. There was also a significant main effect of number magnitude, $F(1,39)=37.91, p<.001, \eta_{\mathrm{p}}{ }^{2}=.493$, with participants responding faster to small numbers $(M=594.61$ 


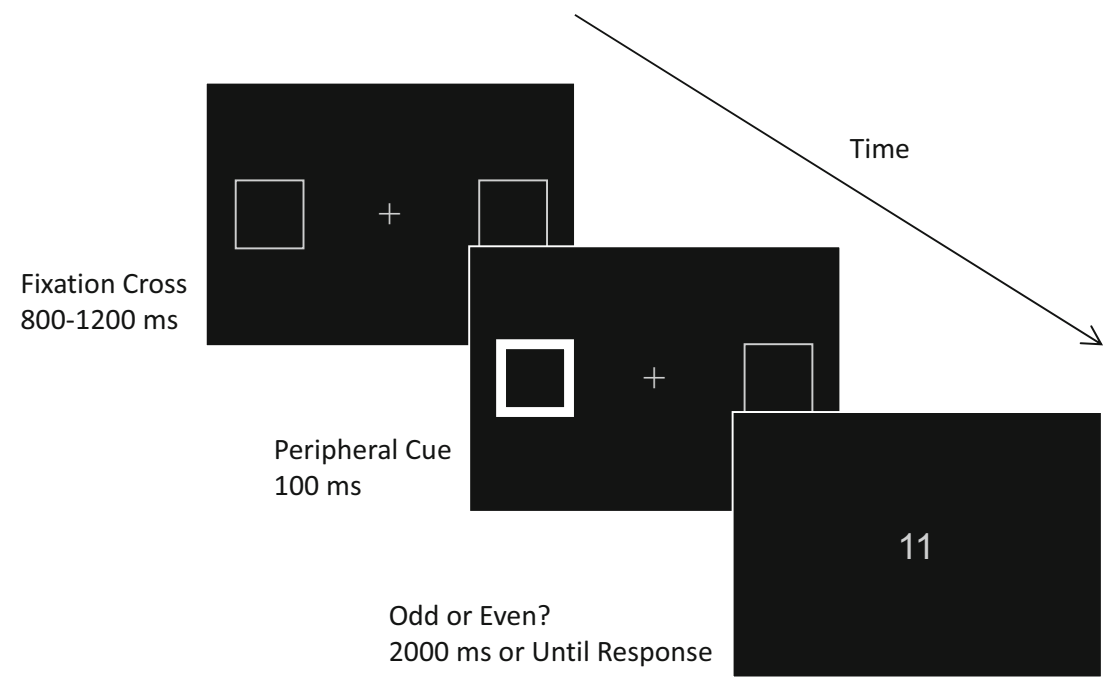

Fig. 1 Example trial sequence in Experiment 1

$\mathrm{ms}, S D=94.39 \mathrm{~ms})$ than to large ones $(M=616.81 \mathrm{~ms}, S D=$ $95.18 \mathrm{~ms}$ ). However, these effects were qualified by a significant three-way interaction between response mapping, cue direction, and number magnitude, $F(1,39)=12.50, p=.001, \eta_{\mathrm{p}}{ }^{2}$ $=.243$. Simple effects tests revealed a significant two-way interaction between response mapping and cue direction for small numbers, $F(1,39)=4.47, p=.041, \eta_{\mathrm{p}}{ }^{2}=.103$, and an opposite two-way interaction between response mapping and cue direction for large numbers, $F(1,39)=10.61, p=.002, \eta_{\mathrm{p}}{ }^{2}=.214$. In both cases, participants responded faster when the cue direction and response side were compatible. Thus, we also observed a Simon effect (Simon, 1969). However, there was no significant two-way interaction between cue direction and number magnitude, $F(1,39)=1.47, p=.232, \eta_{\mathrm{p}}^{2}=.036$. A Bayes factor analysis (Rouder, Morey, Verhagen, Swagman, \& Wagenmakers, 2017) indicated that the null hypothesis was 4.47 times more likely to account for the observed data than the alternative hypothesis that there would be a two-way interaction between cue direction and number magnitude. No other effects were significant (all $p \mathrm{~s} \geq .239$ ). Together, these results suggest that shifts of attention did not influence parity judgments (see Fig. 2a).

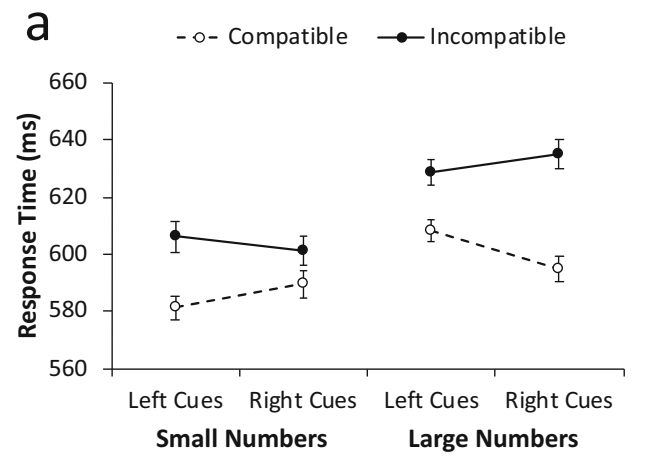

To further test whether shifts of attention influence parity judgments, we also analyzed average error rates using a 2 (response mapping: compatible, incompatible) $\times 2$ (cue direction: left, right) $\times 2$ (number magnitude: small, large) repeated-measures ANOVA. Again, there was a significant main effect of response mapping, $F(1,39)=37.83, p<.001$, $\eta_{\mathrm{p}}{ }^{2}=.492$, with participants making fewer errors when the number magnitude and response side were compatible $(M=$ $3.32 \%, S D=2.88 \%)$ than when they were incompatible $(M=$ $6.83 \%, S D=4.73 \%$ ). Thus, we again observed a responsebased SNARC effect. There was also a significant main effect of cue direction, $F(1,39)=5.55, p=.024, \eta_{\mathrm{p}}{ }^{2}=.125$, with participants making fewer errors when numbers were preceded by leftward cues $(M=4.74 \%, S D=3.36 \%)$ compared to rightward ones $(M=5.41 \%, S D=3.81 \%)$, and a significant main effect of number magnitude, $F(1,39)=13.69, p=.001$, $\eta_{\mathrm{p}}{ }^{2}=.260$, with participants making fewer errors when responding to small numbers $(M=4.41 \%, S D=3.17 \%)$ compared to large ones $(M=5.74 \%, S D=4.09 \%)$. However, there was no significant two-way interaction between cue direction and number magnitude, $F(1,39)=0.74, p=.396, \eta_{\mathrm{p}}{ }^{2}=.019$. A Bayes factor analysis indicated that the null hypothesis was

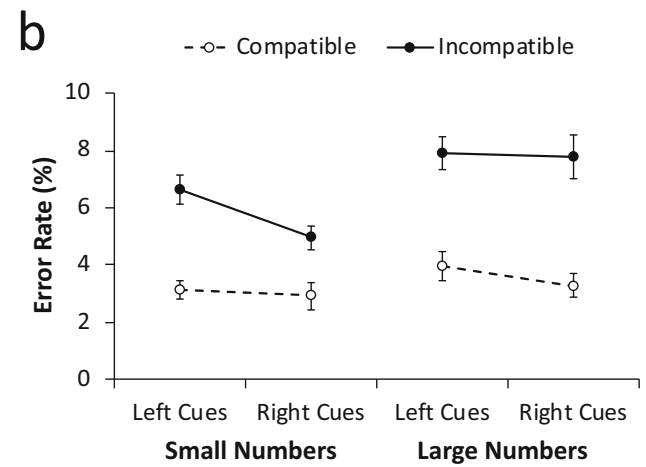

Fig. 2 a Average response times in Experiment 1. b Average error rates in Experiment 1. Error bars in both panels reflect \pm 1 within-subjects standard error (Cousineau, 2005) 
4.54 times more likely to account for the observed data than the alternative hypothesis that there would be a two-way interaction between cue direction and number magnitude. No other effects were significant (all $p \mathrm{~s} \geq .084$ ). Again, these results suggest that shifts of attention did not influence parity judgments (see Fig. 2b).

\section{Discussion}

In Experiment 1, we found no evidence that shifts of attention influenced numerical processing. As predicted, we observed a response-based SNARC effect, with participants responding faster and making fewer errors when the number magnitude and response side were compatible (Dehaene et al., 1993; Dehaene et al., 1990). This suggests that numbers and space share a common representation at the response level. We also observed a Simon effect, with participants responding faster when the cue direction and response side were compatible (Simon, 1969). This is consistent with previous evidence, which suggests that the Simon effect can occur relative to a spatial shift of attention (Nicoletti \& Umiltà, 1994; Rubichi, Nicoletti, Iani, \& Umiltà, 1997; but see Hommel, 1993). However, participants did not respond faster when the cue direction and number magnitude were compatible. Together, these findings suggest that shifts of attention do not influence numerical processing.

\section{Experiment 2}

In Experiment 1, we found no evidence that shifts of attention influenced numerical processing. However, it is possible that shifts of attention only influence numerical processing when the association between numbers and space is relatively explicit. For example, several studies have only replicated the attentional SNARC effect when observers are asked to imagine the position of a number along the mental number line (Fattorini et al., 2015; Pinto et al., 2018) or when the numbers can be mapped onto a consistent spatial structure, such as a line or a clock face (Ristic et al., 2006). In Experiment 2, we attempted to make the association between numbers and space more explicit by presenting a line of six placeholders and cueing the location of the leftmost or rightmost placeholder. We reasoned that by presenting a line of placeholders, the numbers could be more easily mapped onto this spatial structure. As in the previous experiment, if attention plays a role in the spatial representation of numbers, leftward cues should facilitate the processing of small numbers and rightward cues should facilitate the processing of large numbers.

\section{Method}

Participants A new group of 40 University of Toronto undergraduates ( 20 females; mean age $=18.8$ years ) participated for $\$ 10$ or course credit. All participants were right-handed and were native English speakers.

Apparatus and stimuli The apparatus and stimuli were identical to those in Experiment 1.

Procedure and design At the beginning of each trial, a gray fixation cross $\left(45.6 \mathrm{~cd} / \mathrm{m}^{2} ; 1^{\circ} \times 1^{\circ}\right)$ appeared in the center of the screen, flanked by six gray placeholders $\left(45.6 \mathrm{~cd} / \mathrm{m}^{2} ; 2^{\circ} \times\right.$ $2^{\circ}$; see Fig. 3). The placeholders were $0.04^{\circ}$ in thickness, and were presented $3^{\circ}, 6^{\circ}$, and $9^{\circ}$ to the left and right of fixation. After a randomly determined interval between $800-1,200 \mathrm{~ms}$, a white peripheral cue $\left(96.5 \mathrm{~cd} / \mathrm{m}^{2} ; 4^{\circ} \times 4^{\circ}\right)$ was presented at the location of the leftmost or rightmost placeholder. This cue was $0.32^{\circ}$ in thickness, and was presented $9^{\circ}$ to the left or right of fixation. After $100 \mathrm{~ms}$, a two-digit number was presented in the center of the screen. Using the index and middle finger of their right hand, participants pressed the "n" or "m" key to identify whether the number was odd or even. To ensure that the association between numbers and space remained relatively explicit, the placeholders remained on the screen. All other details of the experimental procedure were identical to those in the previous experiment.

\section{Results}

Incorrect responses and response times less than $100 \mathrm{~ms}$ or greater than 2,000 ms were excluded from analysis. To test whether shifts of attention influence parity judgments, we analyzed average response times using a 2 (response mapping: compatible, incompatible) $\times 2$ (cue direction: left, right) $\times 2$ (number magnitude: small, large) repeated-measures ANOVA. The analysis revealed a significant main effect of response mapping, $F(1,39)=40.22, p<.001, \eta_{\mathrm{p}}{ }^{2}=.508$, with participants responding faster when the number magnitude and response side were compatible $(M=622.78 \mathrm{~ms}, S D=102.50$ $\mathrm{ms})$ than when they were incompatible $(M=649.66 \mathrm{~ms}, S D=$ $109.59 \mathrm{~ms}$ ). Thus, as in the previous experiment, we observed a response-based SNARC effect. There was also a significant main effect of cue direction, $F(1,39)=4.60, p=.038, \eta_{\mathrm{p}}{ }^{2}=$ .105 , with participants responding faster when numbers were preceded by rightward cues $(M=633.81 \mathrm{~ms}, S D=103.01 \mathrm{~ms})$ compared to leftward ones $(M=638.64 \mathrm{~ms}, S D=107.91 \mathrm{~ms})$, and a significant main effect of number magnitude, $F(1,39)=$ $18.87, p<.001, \eta_{\mathrm{p}}{ }^{2}=.326$, with participants responding faster to small numbers $(M=629.23 \mathrm{~ms}, S D=103.08 \mathrm{~ms})$ than to large ones $(M=643.22 \mathrm{~ms}, S D=108.33 \mathrm{~ms})$. In addition to these effects, there was a significant two-way interaction between response mapping and number magnitude, $F(1,39)=$ 


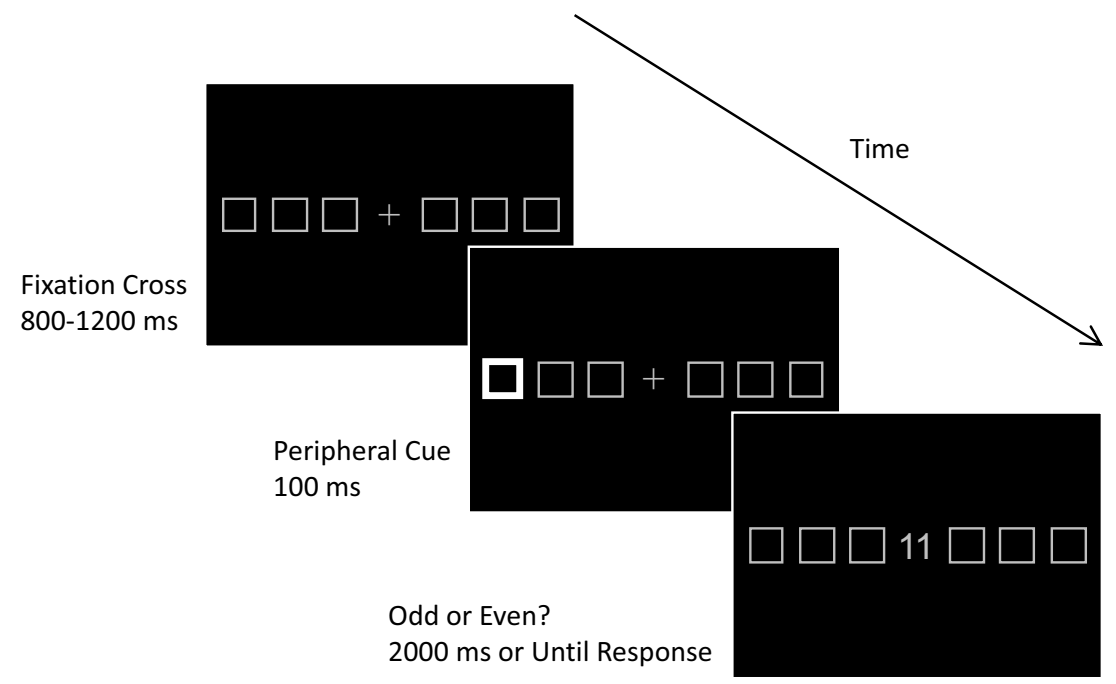

Fig. 3 Example trial sequence in Experiment 2

$7.90, p=.008, \eta_{\mathrm{p}}^{2}=.168$, with participants responding even faster to small numbers when the number magnitude and response side were incompatible. However, these effects were qualified by a significant three-way interaction between response mapping, cue direction, and number magnitude, $F(1$, 39) $=10.04, p=.003, \eta_{\mathrm{p}}{ }^{2}=.243$. Simple effects tests revealed a significant two-way interaction between response mapping and cue direction for small numbers, $F(1,39)=8.32, p=$ $.006, \eta_{\mathrm{p}}{ }^{2}=.176$, and a marginally significant two-way interaction between response mapping and cue direction for large numbers, $F(1,39)=3.60, p=.065, \eta_{\mathrm{p}}{ }^{2}=.085$. In both cases, participants responded faster when the cue direction and response side were compatible. Thus, we observed a Simon effect. However, there was no significant two-way interaction between cue direction and number magnitude, $F(1,39)=$ $0.027, p=.871, \eta_{\mathrm{p}}{ }^{2}=.001$. A Bayes factor analysis indicated that the null hypothesis was 6.22 times more likely to account for the observed data than the alternative hypothesis that there would be a two-way interaction between cue direction and number magnitude. No other effects were significant (all $p \mathrm{~s} \geq$ .244). Together, these results suggest that shifts of attention did not influence parity judgments (see Fig. 4a).

To further test whether shifts of attention influence parity judgments, we also analyzed average error rates using a 2 (response mapping: compatible, incompatible) $\times 2$ (cue direction: left, right) $\times 2$ (number magnitude: small, large) repeated-measures ANOVA. The analysis revealed a significant main effect of response mapping, $F(1,39)=22.21$, $p<.001, \eta_{\mathrm{p}}{ }^{2}=.363$, with participants making fewer errors when the number magnitude and response side were compatible $(M=3.86 \%, S D=4.20 \%)$ than when they were incompatible $(M=6.88 \%, S D=5.51 \%)$. Thus, we again observed a response-based SNARC effect. There was also a significant main effect of number magnitude, $F(1,39)=$ $4.79, p=.035, \eta_{\mathrm{p}}{ }^{2}=.109$, with participants making fewer errors when responding to small numbers $(M=5.03 \%, S D$ $=4.38 \%)$ compared to large ones $(M=5.70 \%, S D=$ $4.74 \%$ ). However, these effects were qualified by a significant three-way interaction between response mapping, cue direction, and number magnitude, $F(1,39)=4.184, p=$ $.048, \eta_{\mathrm{p}}{ }^{2}=.097$. Simple effects tests revealed a marginally significant two-way interaction between response mapping and cue direction for small numbers, $F(1,39)=3.831, p=$ $.057, \eta_{\mathrm{p}}{ }^{2}=.089$, but no significant two-way interaction between response mapping and cue direction for large numbers, $F(1,39)=2.26, p=.140, \eta_{\mathrm{p}}{ }^{2}=.055$. In both cases, participants made fewer errors when the cue direction and response side were compatible. Thus, we again observed a Simon effect. However, there was no significant two-way interaction between cue direction and number magnitude, $F(1,39)=0.13, p=.724, \eta_{\mathrm{p}}{ }^{2}=.003$. A Bayes factor analysis indicated that the null hypothesis was 6.20 times more likely to account for the observed data than the alternative hypothesis that there would be a twoway interaction between cue direction and number magnitude. No other effects were significant (all $p \mathrm{~s} \geq .197$ ). Again, these results suggest that shifts of attention did not influence parity judgments (see Fig. 4b).

\section{Discussion}

In Experiment 2, we again found no evidence that shifts of attention influenced numerical processing. As in the previous experiment, we observed a response-based SNARC effect, with participants responding faster and making fewer errors when the number magnitude and response side were 

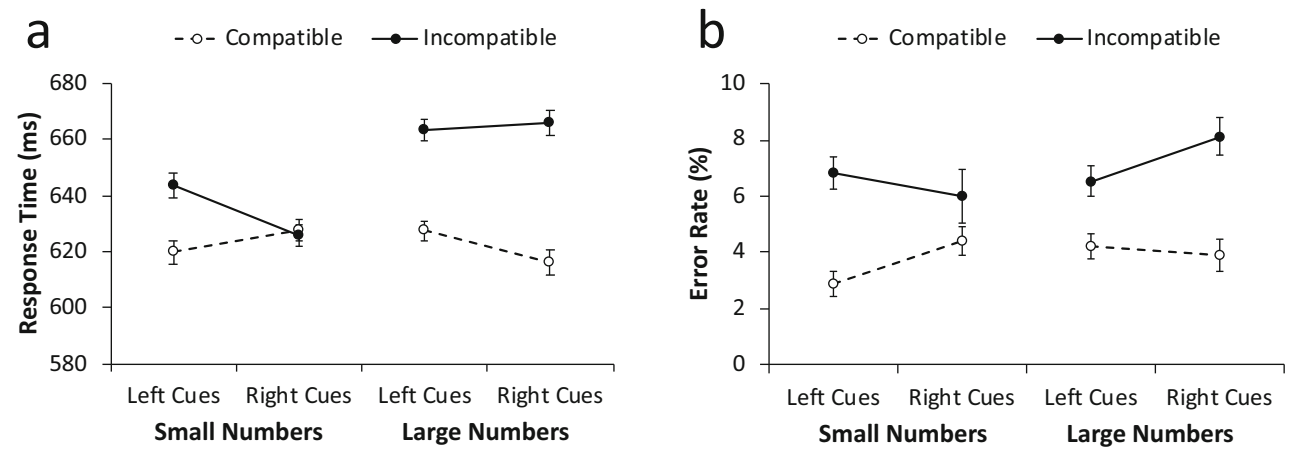

Fig. 4 a Average response times in Experiment 2. b Average error rates in Experiment 2. Error bars in both panels reflect \pm 1 within-subjects standard error (Cousineau, 2005)

compatible (Dehaene et al., 1993; Dehaene et al., 1990). Again, this suggests that numbers and space share a common representation at the response level. We also observed a Simon effect, with participants responding faster and making fewer errors when the cue direction and response side were compatible (Simon, 1969). However, participants did not respond faster when the cue direction and number magnitude were compatible. Together, these findings suggest that shifts of attention do not influence numerical processing, even when the association between numbers and space is relatively explicit.

\section{Experiment 3}

In Experiment 2, we again found no evidence that shifts of attention influenced numerical processing. However, it is possible that shifts of attention only influence numerical processing when number magnitude is directly relevant to observers' task. For example, several studies have only replicated the attentional SNARC effect when observers are asked to remember (Casarotti et al., 2007) or judge the magnitude of a number (Zanolie \& Pecher, 2014; but see Fattorini et al., 2015; Pinto et al., 2018). In Experiment 3, we made number magnitude directly relevant to participants' task by having participants judge whether the number is smaller or larger than 55 . As in the previous experiments, if attention plays a role in the spatial representation of numbers, leftward cues should facilitate the processing of small numbers and rightward cues should facilitate the processing of large numbers.

\section{Method}

Participants A new group of 45 University of Toronto undergraduates ( 33 females; mean age $=20.6$ years) participated for $\$ 10$ or course credit. All participants were right-handed and were native English speakers. Five participants were excluded because they made a large proportion of errors $(\sim 35 \%$ or greater).

Apparatus and stimuli The apparatus and stimuli were identical to those in the previous experiments.

Procedure and design The task was the same as in Experiment 2 , with the exception that participants judged whether the number was smaller or larger than 55. All other details of the experimental procedure were identical to those in the previous experiment.

\section{Results}

Incorrect responses and response times less than $100 \mathrm{~ms}$ or greater than 2,000 ms were excluded from analysis. To test whether shifts of attention influence magnitude judgments, we analyzed average response times using a 2 (response mapping: compatible, incompatible) $\times 2$ (cue direction: left, right $) \times 2$ (number magnitude: small, large) mixed-model ANOVA. Response mapping was entered as a between-subjects variable, and cue direction and number magnitude were entered as within-subjects variables. The analysis revealed a significant main effect of number magnitude, $F(1,38)=12.92, p=$ $.001, \eta_{\mathrm{p}}{ }^{2}=.254$, with participants responding faster to large numbers ( $M=551.87 \mathrm{~ms}, S D=96.16 \mathrm{~ms}$ ) than to small ones $(M=568.40 \mathrm{~ms}, S D=102.01 \mathrm{~ms})$. There was also a significant two-way interaction between response mapping and number magnitude, $F(1,38)=5.04, p=.031, \eta_{\mathrm{p}}{ }^{2}=.117$, with participants responding even faster to large numbers when the number magnitude and response side were compatible. However, these effects were qualified by a significant threeway interaction between response mapping, cue direction, and number magnitude, $F(1,38)=62.45, p<.001, \eta_{\mathrm{p}}{ }^{2}=.622$. Simple effects tests revealed a significant two-way interaction between response mapping and cue direction for small numbers, $F(1,38)=35.51, p<.001, \eta_{\mathrm{p}}{ }^{2}=.483$, and an opposite 
two-way interaction between response mapping and cue direction for large numbers, $F(1,38)=25.58, p<.001, \eta_{\mathrm{p}}{ }^{2}=$ .402. In both cases, participants responded faster when the cue direction and response side were compatible. Thus, we observed a Simon effect. However, there was no significant two-way interaction between cue direction and number magnitude, $F(1,38)=0.03, p=.869, \eta_{\mathrm{p}}{ }^{2}=.001$. A Bayes factor analysis indicated that the null hypothesis was 4.19 times more likely to account for the observed data than the alternative hypothesis that there would be a two-way interaction between cue direction and number magnitude. No other effects were significant (all $p \mathrm{~s} \geq .369$ ). Together, these results suggest that shifts of attention did not influence magnitude judgments (see Fig. 5a).

To further test whether shifts of attention influence magnitude judgments, we also analyzed average error rates using a 2 (response mapping: compatible, incompatible) $\times 2$ (cue direction: left, right) $\times 2$ (number magnitude: small, large) mixed-model ANOVA. Response mapping was entered as a between-subjects variable, and cue direction and number magnitude were entered as within-subjects variables. Again, the analysis revealed a significant three-way interaction between response mapping, cue direction, and number magnitude, $F(1,38)=18.10, p<.001, \eta_{\mathrm{p}}{ }^{2}=$ .323. Simple effects tests revealed a significant two-way interaction between response mapping and cue direction for small numbers, $F(1,38)=14.57, p<.001, \eta_{\mathrm{p}}{ }^{2}=.277$, and a marginally significant two-way interaction between response mapping and cue direction for large numbers, $F(1,38)=3.77, p=.060, \eta_{\mathrm{p}}{ }^{2}=$ .090. In both cases, participants made fewer errors when the cue direction and response side were compatible. Thus, we again observed a Simon effect. However, there was no significant two-way interaction between cue direction and number magnitude, $F(1,38)=0.13, p=.723, \eta_{\mathrm{p}}^{2}=.003$. A Bayes factor analysis indicated that the null hypothesis was 4.67 times more likely to account for the observed data than the alternative hypothesis that there would be a two-way interaction between cue direction and number magnitude. No other effects were significant (all $p \mathrm{~s} \geq$ .075). Again, these results suggest that shifts of attention did not influence magnitude judgments (see Fig. 5b).

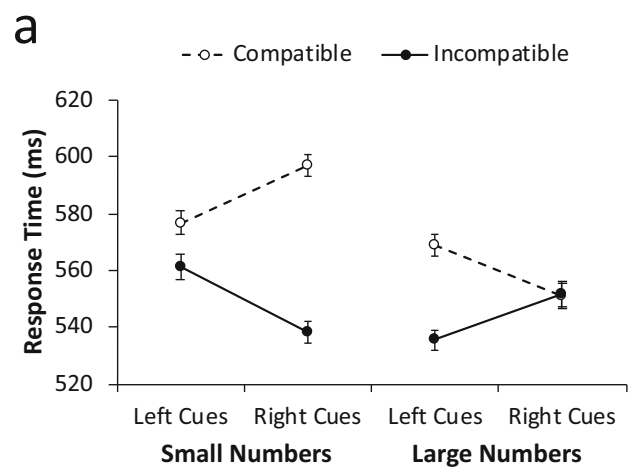

\section{Discussion}

In Experiment 3, we again found no evidence that shifts of attention influenced numerical processing. Unlike the previous experiments, we failed to observe a response-based SNARC effect. However, we observed a large Simon effect, with participants responding faster and making fewer errors when the cue direction and response side were compatible (Simon, 1969). As in the previous experiments, participants did not respond faster when the cue direction and number magnitude were compatible. Together, these findings suggest that shifts of attention do not influence numerical processing, even when number magnitude is directly relevant to observers' task.

\section{General discussion}

In the present study, we assessed whether shifts of attention can influence numerical processing. Participants viewed a leftward or rightward peripheral cue followed by a centrally presented number, then judged whether the number was odd or even. Participants responded faster and made fewer errors when the number magnitude and response side were compatible, revealing a response-based SNARC effect (Dehaene et al., 1993; Dehaene et al., 1990). Participants also responded faster when the cue direction and response side were compatible, revealing a Simon effect (Simon, 1969). However, participants did not respond faster when the cue direction and number magnitude were compatible. This suggests that shifts of attention did not influence numerical processing. Similar findings were observed when the association between numbers and space was relatively explicit. Moreover, although we failed to observe a response-based SNARC effect when number magnitude was directly relevant to observers' task, we observed a large Simon effect. Together, these findings suggest that although numbers and space share a common representation at the response level, attention does not play a substantial role in the spatial representation of numbers.

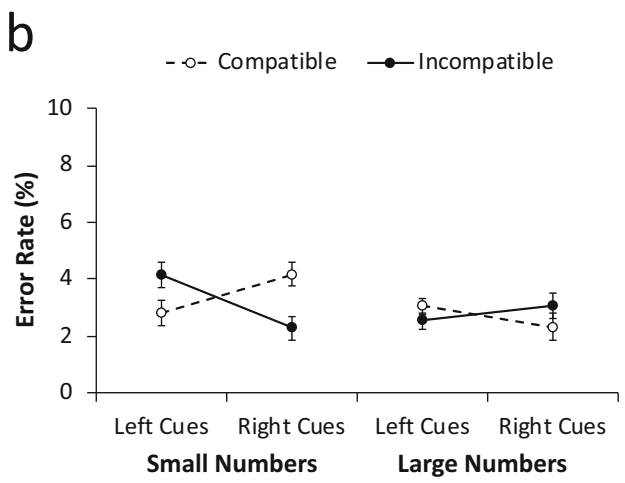

Fig. 5 a Average response times in Experiment 3. b Average error rates in Experiment 3. Error bars in both panels reflect \pm 1 within-subjects standard error (Cousineau, 2005) 
The present findings provide a clear challenge to the broader claim that shifts of attention underlie the processing of numerical information. Based on neurophysiological evidence, several influential theories of numerical cognition have proposed that shifts of attention directly influence numerical processing (Dehaene et al., 2003; Hubbard et al., 2005). However, while numbers, space, and attention rely on many of the same areas in posterior parietal cortex, this does not mean that attention plays a causal role in the spatial representation of numbers. Instead, it is possible that numbers and attention share overlapping neural resources based on their common reliance on spatial information. According to this explanation, while a common spatial representation underlies both shifts of attention and numerical processing, shifts of attention do not directly influence numerical processing. Such an explanation can account for many findings in the numerical cognition literature. For example, although patients with right parietal damage show similar impairments when bisecting a line or judging the midpoint of a numerical interval (Zorzi et al., 2002), these findings may reflect damage to the common spatial representation that underlies both shifts of attention and numerical processing.

Importantly, the present findings also add to a growing body of research that has questioned whether viewing numbers can produce shifts of attention. A number of studies have either failed to replicate the attentional SNARC effect (Bonato et al., 2009; Colling et al., 2020; Pellegrino et al., 2019; see also Ranzini et al., 2009; Salillas et al., 2008; Schuller et al., 2015) or have only replicated this effect under certain conditions. For example, several studies have only replicated the attentional SNARC effect when observers are asked to imagine the position of a number along the mental number line (Fattorini et al., 2015; Pinto et al., 2018; see also Ristic et al., 2006) or when observers are asked to judge the magnitude of a number (Zanolie \& Pecher, 2014; see also Casarotti et al., 2007; but see Fattorini et al., 2015; Pinto et al., 2018). In the present study, we found no evidence that shifts of attention influence numerical processing, even under conditions which have been used to replicate the attentional SNARC effect. This suggests that attention does not play a substantial role in the spatial representation of numbers. If this is the case, it is possible that viewing numbers may only produce spatial shifts of attention when these processes require access to a common spatial representation.

Although we found no evidence that shifts of attention influence numerical processing, the present findings are consistent with many theoretical models, which suggest that numbers and space share a common representation at the response level (Daar \& Pratt, 2008; Gevers et al., 2005; Gevers et al., 2006; Keus \& Schwarz, 2005; Keus et al., 2005). For example, several studies have found that the SNARC effect interacts with the Simon effect (Gevers et al., 2005; Keus \& Schwarz, 2005; but see Mapelli et al., 2003), suggesting that these effects occur at a similar processing stage. There is also evidence that viewing numbers can directly influence response selection (Daar \& Pratt, 2008). However, the SNARC and attentional SNARC effects do not appear to interact with one another (Fattorini et al., 2015). In the present study, we found no evidence that shifts of attention influence numerical processing. However, we observed both a response-based SNARC effect and a Simon effect. Because both effects are thought to occur at the response selection stage, this provides further evidence that numbers and space share a common representation at the response level.

While the present findings are consistent with the notion that shifts of attention do not influence numerical processing, several aspects of these findings warrant further discussion. First, although we observed both a response-based SNARC effect and a Simon effect in Experiments 1 and 2, these effects did not appear to interact. This differs from several previous studies, which have found that the SNARC effect interacts with the Simon effect (Gevers et al., 2005; Keus \& Schwarz, 2005; but see Mapelli et al., 2003). However, the present study differs from these studies in one important way. In the previous studies, participants viewed a number that was presented to the left or right of fixation. However, in the present study, participants viewed a leftward or rightward peripheral cue followed by a centrally presented number. It is possible that when the cue and number are presented at different spatial locations, the SNARC and Simon effects do not strongly interact. Previous evidence suggests that the Simon effect can occur relative to a spatial shift of attention (Nicoletti \& Umiltà, 1994; Rubichi et al., 1997; but see Hommel, 1993). In such cases, the Simon effect may be relatively independent of the SNARC effect.

Second, although we observed a response-based SNARC effect in Experiments 1 and 2, we failed to observe this effect in Experiment 3. However, we observed a large Simon effect in this experiment. One possible explanation for these findings is that shifts of attention disrupt the association between numbers and space when number magnitude is directly relevant to observers' task. Consistent with this explanation, Irwin and Thomas (2007) found that saccadic eye movements interfered with numerical processing when observers were asked to judge the magnitude of a number. However, this effect was only observed for leftward saccades. A more plausible explanation is that the SNARC and Simon effects interact more strongly when number magnitude is directly relevant to observers' task. Consistent with this explanation, Gevers et al. (2005) found that the SNARC effect strongly interacted with the Simon effect when observers were asked to judge the magnitude of a number. Such an explanation can account for both our failure to observe a response-based SNARC effect in Experiment 3 and the large Simon effect observed in this experiment. 
Third, although the present findings suggest that attention does not play a substantial role in the spatial representation of numbers, it is possible that shifts of attention can influence numerical processing under certain conditions. For example, Stoianov, Kramer, Umiltà, and Zorzi (2008) had participants view a centrally presented number followed by a leftward or rightward peripheral cue, then asked them to judge the parity or magnitude of the number. In this case, leftward cues facilitated the processing of small numbers and rightward cues facilitated the processing of large numbers. However, a similar effect was not observed when the cue preceded the number (see also Kramer, Stoianov, Umiltà, \& Zorzi, 2011). Based on these findings, it is possible that shifts of attention may play a limited role in the spatial representation of numbers. However, because these findings were only observed when the cue followed the number, it is unlikely that shifts of attention directly influenced numerical processing. Moreover, because participants verbally judged the parity or magnitude of the number, the researchers could not test whether numbers and space share a common representation at the response level. Future work should attempt to clarify these findings and identify the conditions under which shifts of attention influence numerical processing.

Lastly, it is worth noting that shifts of attention can influence the processing of other stimuli with implicit spatial associations. For example, a number of studies have suggested that viewing words with upward or downward spatial associations can produce spatial shifts of attention (Chasteen, Burdzy, \& Pratt, 2010; Estes, Verges, \& Barsalou, 2008; Gozli, Chasteen, \& Pratt, 2013). More recently, Mills et al. (2018) found that shifts of attention can influence the processing of these words. Why does attention play a role in the spatial representation of words but not numbers? According to theories of embodied cognition, the representation of many concepts may be grounded in representations of space (Barsalou, 1999; Lakoff \& Johnson, 1980, 1999). If this is the case, viewing words with upward or downward spatial associations may automatically activate the corresponding spatial representations, which may in turn may produce spatial shifts of attention. Some researchers have also suggested that the spatial grounding of concepts may be bidirectional (e.g., Mills et al., 2018). If this is the case, shifts of attention may directly influence language processing. Future work should attempt to clarify why attention plays a role in the spatial representation of words but not numbers.

In summary, we found no evidence that shifts of attention influence numerical processing. Although we observed both a response-based SNARC effect and a Simon effect, participants did not respond faster when the cue direction and number magnitude were compatible. Similar findings were observed when the association between numbers and space was relatively explicit or when number magnitude was directly relevant to observers' task. Together, these findings challenge the broader assumption that shifts of attention underlie the processing of numerical information, and suggest one possible reason why viewing numbers does not automatically produce spatial shifts of attention. Specifically, if attention does not play a substantial role in the spatial representation of numbers, viewing numbers may only produce spatial shifts of attention when these processes require access to a common spatial representation.

Acknowledgements This research was supported by an NSERC Discovery Grant (2016-06359) to Jay Pratt.

Open practices statement The materials, analyses, and data from all of our experiments are available on the Open Science Framework (https:// osf.io/264y7/). None of our experiments were preregistered.

\section{References}

Barsalou, L. W. (1999). Perceptual symbol systems. Behavioral and Brain Sciences, 22(4), 577-609.

Bonato, M., Priftis, K., Marenzi, R., \& Zorzi, M. (2009). Normal and impaired reflexive orienting of attention after central nonpredictive cues. Journal of Cognitive Neuroscience, 21(4), 745-759.

Casarotti, M., Michielin, M., Zorzi, M., \& Umiltà, C. (2007). Temporal order judgment reveals how number magnitude affects visuospatial attention. Cognition, 102(1), 101-117.

Chasteen, A. L., Burdzy, D. C., \& Pratt, J. (2010). Thinking of God moves attention. Neuropsychologia, 48(2), 627-630.

Colby, C. L., \& Goldberg, M. E. (1999). Space and attention in parietal cortex. Annual Review of Neuroscience, 22, 319-349.

Colling, L. J., Szücs, D., De Marco, D., Cipora, K., Ulrich, R., Nuerk, H.C., ... McShane, B. B. (2020). Registered replication report on Fischer, Castel, Dodd, and Pratt (2003). Advances in Methods and Practices in Psychological Science, 3(2), 143-162.

Cousineau, D. (2005). Confidence intervals in within-subject designs: A simpler solution to Loftus and Masson's method. Tutorials in Quantitative Methods for Psychology, 1(1), 42-45.

Daar, M., \& Pratt, J. (2008). Digits affect actions: The SNARC effect and response selection. Cortex, 44(4), 400-405.

Dehaene, S., Bossini, S., \& Giraux, P. (1993). The mental representation of parity and number magnitude. Journal of Experimental Psychology: General, 122(3), 371-396.

Dehaene, S., Dupoux, E., \& Mehler, J. (1990). Is numerical comparison digital? Analogical and symbolic effects in two-digit number comparison. Journal of Experimental Psychology: Human Perception and Performance, 16(3), 626-641.

Dehaene, S., Piazza, M., Pinel, P., \& Cohen, L. (2003). Three parietal circuits for number processing. Cognitive Neuropsychology, 20(3), 487-506.

Dodd, M. D., Van der Stigchel, S., Leghari, M. A., Fung, G., \& Kingstone, A. (2008). Attentional SNARC: There's something special about numbers (let us count the ways). Cognition, 108(3), 810 818.

Estes, Z., Verges, M., \& Barsalou, L. W. (2008). Head up, foot down: Object words orient attention to the objects' typical location. Psychological Science, 19(2), 93-97.

Fattorini, E., Pinto, M., Rotondaro, F., \& Doricchi, F. (2015). Perceiving numbers does not cause automatic shifts of spatial attention. Cortex, 73, 298-316.

Faul, F., Erdfelder, E., Lang, A.-G., \& Buchner, A. (2007). G*Power 3: A flexible statistical power analysis program for the social, behavioral, 
and biomedical sciences. Behavioral Research Methods, 39(2), 175-191.

Fischer, M. H. (2003). Spatial representations in number processing: Evidence from a pointing task. Visual Cognition, 10(4), 493-508.

Fischer, M. H., Castel, A. D., Dodd, M. D., \& Pratt, J. (2003). Perceiving numbers causes spatial shifts of attention. Nature Neuroscience, 6(6), 555-556.

Fischer, M. H., Warlop, N., Hill, R. L., \& Fias, W. (2004). Oculomotor bias induced by number perception. Experimental Psychology, 51(2), 91-97.

Galfano, G., Rusconi, E., \& Umiltà, C. (2006). Number magnitude orients attention, but not against one's will. Psychonomic Bulletin \& Review, 13(5), 869-874.

Gevers, W., Caessens, B., \& Fias, W. (2005). Towards a common processing architecture underlying Simon and SNARC effects. European Journal of Cognitive Psychology, 17(5), 659-673.

Gevers, W., Verguts, T., Reynvoet, B., Caessens, B., \& Fias, W. (2006). Numbers and space: A computational model of the SNARC effect. Journal of Experimental Psychology: Human Perception and Performance, 32(1), 32-44.

Gozli, D. G., Chasteen, A. L., \& Pratt, J. (2013). The cost and benefit of implicit spatial cues for visual attention. Journal of Experimental Psychology: General, 142(4), 1028-1046.

He, D., He, X., Zhao, T., Wang, J., Li, L., \& Louwerse, M. (2020). Does number perception cause automatic shifts of spatial attention? A study of the Att-SNARC effect in numbers and Chinese months. Frontiers in Psychology, 11, 1-13.

Hommel, B. (1993). The role of attention for the Simon effect. Psychological Research, 55(3), 208-222.

Hubbard, E. M., Piazza, M., Pinel, P., \& Dehaene, S. (2005). Interactions between number and space in parietal cortex. Nature Reviews Neuroscience, 6(6), 435-448.

Irwin, D. E., \& Thomas, L. E. (2007). The effect of saccades on number processing. Perception \& Psychophysics, 69(3), 450-458.

Keus, I. M., Jenks, K. M., \& Schwarz, W. (2005). Psychophysiological evidence that the SNARC effect has its functional locus in a response selection stage. Cognitive Brain Research, 24(1), 48-56.

Keus, I. M., \& Schwarz, W. (2005). Searching for the functional locus of the SNARC effect: Evidence for a response-related origin. Memory \& Cognition, 33(4), 681-695.

Kramer, P., Stoianov, I., Umiltà, C., \& Zorzi, M. (2011). Interactions between perceptual and numerical space. Psychonomic Bulletin \& Review, 18(4), 722-728.

Lakoff, G., \& Johnson, M. (1980). Metaphors we live by. Chicago, IL: University of Chicago Press.

Lakoff, G., \& Johnson, M. (1999). Philosophy in the flesh: The embodied mind and its challenge to Western thought. New York, NY: Basic Books.

Mapelli, D., Rusconi, E., \& Umiltà, C. (2003). The SNARC effect: An instance of the Simon effect? Cognition, 88(3), B1-B10.

Mills, M. Boychuk, P., Chasteen, A. L., \& Pratt, J. (2018). Attention goes both ways: Shifting attention influences lexical decisions. Journal of Experimental Psychology: General, 147(2), 282-291.

Nicoletti, R., \& Umiltà, C. (1994). Attention shifts produce spatial stimulus codes. Psychological Research, 56(3), 144-150.
Nuerk, H.-C., Weger, U., \& Willmes, K. (2001). Decade breaks in the mental number line? Putting the tens and units back in different bins. Cognition, 82(1), B25-B33.

Pellegrino, M., Pinto, M., Marson, F., Lasaponara, S., Rossi-Arnaud, C., Cestari, V., \& Doricchi, F. (2019). The Attentional-SNARC effect 16 years later: No automatic space-number association (taking into account finger counting style, imagery vividness, and learning style in 174 participants). Experimental Brain Research, 237(10), 26332643.

Pinto, M., Fattorini, E., Lasaponara, S., D’Onofrio, M., Fortunato, G., \& Doricchi, F. (2018). Visualising numerals: An ERPs study with the attentional SNARC task. Cortex, 101, 1-15.

Posner, M. I. (1980). Orienting of attention. Quarterly Journal of Experimental Psychology, 32(1), 3-25.

Proctor, R. W., \& Cho, Y. S. (2006). Polarity correspondence: A general principle for performance of speeded binary classification tasks. Psychological Bulletin, 132(3), 416-442.

Ranzini, M., Dehaene, S., Piazza, M., \& Hubbard, E. M. (2009). Neural mechanisms of attentional shifts due to irrelevant spatial and numerical cues. Neuropsychologia, 47(12), 2615-2624.

Ristic, J., Wright, A., \& Kingstone, A. (2006). The number line effect reflects top-down control. Psychonomic Bulletin \& Review, 13(5), $862-868$.

Rouder, J. N., Morey, R. D., Verhagen, J., Swagman, A. R., \& Wagenmakers, E.-J. (2017). Bayesian analysis of factorial designs. Psychological Methods, 22(2), 304-321.

Rubichi, S., Nicoletti, R., Iani, C., \& Umiltà, C. (1997). The Simon effect occurs relative to the direction of an attention shift. Journal of Experimental Psychology: Human Perception and Performance, 23(5), 1353-1364.

Salillas, E., El Yagoubi, R., \& Semenza, C. (2008). Sensory and cognitive processes of shifts of spatial attention induced by numbers: An ERP study. Cortex, 44(4), 406-413.

Schuller, A.-M., Hoffman, D., Goffaux, V., \& Schiltz, C. (2015). Shifts of spatial attention cued by irrelevant numbers: Electrophysiological evidence from a target discrimination task. Journal of Cognitive Psychology, 27(4), 442-458.

Schwarz, W., \& Keus, I. M. (2004). Moving the eyes along the mental number line: Comparing SNARC effects with saccadic and manual responses. Perception \& Psychophysics, 66(4), 651-664.

Schwarz, W., \& Müller, D. (2006). Spatial associations in number-related tasks: A comparison of manual and pedal responses. Experimental Psychology, 53(1), 4-15.

Simon, J. R. (1969). Reactions toward the source of stimulation. Journal of Experimental Psychology, 81(1), 174-176.

Stoianov, I., Kramer, P., Umiltà, C., \& Zorzi, M. (2008). Visuospatial priming of the mental number line. Cognition, 106(2), 770-779.

Zanolie, K., \& Pecher, D. (2014). Number-induced shifts in spatial attention: A replication study. Frontiers in Psychology, 5, 1-10.

Zorzi, M., Priftis, K., \& Umiltà, C. (2002). Neglect disrupts the mental number line. Nature, 417(6885), 138-139.

Publisher's note Springer Nature remains neutral with regard to jurisdictional claims in published maps and institutional affiliations. 